Notre Dame Journal of Formal Logic Volume 23, Number 1, January 1982

\title{
Elementary Formal Systems as a Framework for Relative Recursion Theory
}

\author{
BRUCE M. HOROWITZ
}

1 Background on elementary formal systems A nonempty ordered finite set $K$ is called an alphabet. Members of this set are called symbols. By a word in $K$, we mean a nonempty finite sequence of symbols of $K$. Given the $n$ symbols $x_{1}, x_{2}, \ldots, x_{n}$ of $K$ (not necessarily distinct), let $x_{1} x_{2} \ldots x_{n}$ be the word in $K$ whose $i^{\text {th }}$ symbol is $x_{i}(1 \leqslant i \leqslant n)$. The length of a word is the number of symbols (counting repetitions) in that word. If $X$ and $Y$ are words in $K$, and $X$ is the word $x_{1} x_{2} \ldots x_{n}, Y$ is the word $y_{1} y_{2} \ldots y_{m}$, then $X Y$ is also a word in $K$ and $X Y$ is the word $x_{1} x_{2} \ldots x_{n} y_{1} y_{2} \ldots y_{m} . X Y$ is called the concatenation of $X$ and $Y$.

Definition of an elementary formal system (EFS) By an elementary formal system $(E)$ over an alphabet $K$, we mean a collection of the following:

1. the alphabet $K$

2. another alphabet of symbols called variables, which range over words in $K$

3. another alphabet of symbols called predicates, each of which is assigned a unique positive integer called its degree

4. two more symbols called the implication sign and the punctuation sign

5. a finite sequence $A_{1}, \ldots, A_{n}$ of strings which are well-formed formulas, called axioms. (The rules for their formation are listed below.)

The alphabets in 1-4 are to be mutually disjoint. Elements of $K$ usually are denoted by ' $a$ ', ' $b$ ', etc., variables by ' $x_{1}$ ', ' $x_{2}$ ', etc., or ' $x$ ', ' $y$ ', etc., and predicates by ' $P$ ', ' $Q$ ', etc., sometimes with superscripts and subscripts. The implication sign and the punctuation sign are denoted, respectively, by ' $\rightarrow$ ' and ','. 
Let $K$ consist of the symbols $a_{1}, \ldots, a_{n}$. By a term $t$ of $(E)$ we mean any finite string $t_{1} \ldots t_{k}$, where each $t_{i}(1 \leqslant i \leqslant k)$ is either some $a_{j}(1 \leqslant j \leqslant n)$ or a variable. By an atomic (well-formed) formula of $(E)$ we mean an expression of the form $P t_{1}, \ldots, t_{n}$, where $P$ is a predicate of degree $n$ and $t_{1}, \ldots, t_{n}$ are terms of $(E)$. By a well-formed formula (wff) $F$ of $(E)$ we mean either an atomic formula $F_{1}$, or an expression of the form $F_{1} \rightarrow F_{2}$, where $F_{1}$ is an atomic formula of $(E)$ and $F_{2}$ is a well-formed formula of $(E)$.

An instance of a wff $X$ is any string obtained from $X$ by substituting words in $K$ for all variables, subject to the condition that the same word is substituted for all occurrences of any variable. A sentence is a wff with no variables.

A string $X$ of $(E)$ is a theorem of $(E)$ if $X$ is either an axiom of $(E)$, or is derivable from the axioms of $(E)$ by a finite number of applications of the following two rules of inference:

Rule I. We may substitute words in $K$ for variables to obtain instances of wffs.

Rule II. Providing $X_{1}$ is atomic, we may infer $X_{2}$ from $X_{1}$ and $X_{1} \rightarrow X_{2}$. (Rule of Modus Ponens.)

If $X$ is a theorem of $(E)$, we write $\vdash_{(E)} X$.

Let $P$ be a predicate of degree 1 in an $\operatorname{EFS}(E)$ over $K$.

Let $A$ be a set of words in $K$. We say that $P$ represents $A$ in $(E)$ if for every word $X$ in $K, X \in A \Longleftrightarrow \digamma_{(E)} P X$.

A set of words in $K$ is called formally representable over $K$ if $A$ is representable in some EFS over $K . A$ is called solvable over $K$ if both $A$ and $\widetilde{A}$ are formally representable over $K$.

There is a corresponding definition for relations: Let $R\left(x_{1}, \ldots, x_{n}\right)$ be an $n$-place relation. We say that $R$ is representable in the $\operatorname{EFS}(E)$ if there is some predicate of degree $n$ in $(E)$ such that $R\left(x_{1}, \ldots, x_{n}\right) \Longleftrightarrow \vdash_{(E)} P x_{1}, \ldots, x_{n}$.

Since a function may be viewed as a single-valued relation, we have a corresponding definition for functions: We say that the function $f\left(x_{1}, \ldots, x_{n}\right)$ is representable in the EFS $(E)$ if the relation $f\left(x_{1}, \ldots, x_{n}\right)=y$ is representable in the $\operatorname{EFS}(E)$.

Let $B=\{0,1\}$. A binary elementary formal system is any EFS $(E)$ over the alphabet $B$. A binary numeral is a word in $B$. The binary numeral $b_{n} b_{n-1} \ldots$ $b_{1} b_{0}$ represents the number $b_{0}+2 b_{1}+4 b_{2}+\ldots+2^{n} b_{n}$.

A set of natural numbers is called recursively enumerable (r.e.) if it is representable in some binary EFS. A set of natural numbers is called recursive if it and its complement are r.e. The same definition holds for relations.

Binary elementary formal systems are the equivalent of Smullyan's dyadic elementary formal systems [4]. However, we prefer the former for certain technical reasons.

2 Pseudoelementary formal systems Let $n$ be a natural number. Let $\bar{n}$ be the binary numeral representing the number $n$. We define special axioms for $B$ to be all axioms of the form $P \bar{n}$ if $n \in B$ and $Q \bar{n}$ if $n \notin B$, where $P$ and $Q$ are two specific unary predicate letters.

We say that a set $A$ is recursively enumerable in a set $B$ if $A$ is representable in some binary EFS $(E)$ having special axioms for $B$, and for $B$ alone. We say $A$ is recursive in $B$ if both $A$ and $\widetilde{A}$ are r.e. in $B$. 
Note that an EFS with special axioms is not a true EFS because the axioms of such an "EFS" must always be infinite in number. Therefore, we call an "EFS" with special axioms for $B$ a pseudoelementary formal system (PEFS). To further illuminate this point, we show that if a PEFS were a genuine EFS, then all sets would be recursive! In [4], Smullyan showed that r.e. by EFS corresponds to the standard usage of r.e. Therefore, let $A$ be any set of natural numbers. Construct the PEFS $(E)$ having as axioms only the special axioms for $A$. In this system $(E), A$ is representable and so is $\widetilde{A}: n \in A \Rightarrow \vdash_{(E)} P \bar{n}$ by axiom. If $\vdash_{(E)} P \bar{n}$, then it must be that $n \in A$, for the only way we could prove $P \bar{n}$ in $(E)$ would be by an axiom. Thus $A$ is representable in $(E)$ by $P$. Similarly, $\widetilde{A}$ is representable in $(E)$ by $Q$. Thus, $A$ is recursive. Since every set is not recursive (or even r.e.) it follows that $(E)$ is not a true EFS.

Note that ' $A$ is r.e. in $\phi$ ' is equivalent to ' $A$ is r.e.'. This is seen as follows: Suppose $A$ is r.e. Then $A$ is representable in some binary EFS $(E)$ by a predicate $P$, i.e., $n \in A \Longleftrightarrow \downarrow_{(E)} P \bar{n}$. Let $Q, R$ be predicates distinct from any predicate in $(E)$. Construct the PEFS $\left(E^{\prime}\right)$ as follows: To the axioms of $(E)$, add the special axioms

\section{$Q \bar{n}$ if $n \in \phi, R \bar{n}$ if $n \notin \phi$.}

(This amounts to adding $R \bar{n}$ for all $n$.) It is easy to see that $P$ still represents $A$ in $\left(E^{\prime}\right)$. Now suppose $A$ is r.e. in $\phi$. Then $A$ is representable in some PEFS $(E)$ with special axioms for $\phi$ of form $Q \bar{n}$ if $n \epsilon \phi$ and $R \bar{n}$ if $n \phi \phi$. Since $n \phi \phi$ for all $n, R \bar{n}$ is a special axiom for all $n$. Thus, we may replace $R \bar{n}$, for all $n$, by the regular axiom $R x$, to obtain the EFS $\left(E^{\prime}\right)$. In $\left(E^{\prime}\right), A$ is represented by the same predicate representing it in $(E)$.

It readily follows that ' $A$ is recursive in $\phi$ ' is equivalent to ' $A$ is recursive'.

The assertion ' $A$ is r.e. in $B$ ' is a formal way of saying that the elements of $A$ may be generated by some mechanical process, assuming one is allowed to use a finite number of facts about membership in $B$. To see this, suppose $A$ is represented by $P$ in a PEFS $(E)$ having special axioms for $B$. Enumerate all proofs in $(E)$, picking $n$ if $P \bar{n}$ happens to be the last line in a proof. This will generate the set $A$. If $n \in A$, then $P \bar{n}$ will eventually turn up as a theorem of $(E)$. However, if $n \notin A$, at any given stage even though $P \bar{n}$ has not turned up, we could never be sure that it won't show up at some future time. Now the reason we are limited to using a finite number of facts about $B$ is that a proof is a finite sequence. Thus, even though an infinite number of special axioms for $B$ are available in $(E)$, only finitely many of them are allowed to occur in any proof in $(E)$. This infinite collection of knowledge about $B$ corresponds to what recursion theorists call an "oracle" for $B$.

The assertion ' $A$ is recursive in $B$ ' is a formal way of saying that one can effectively decide whether or not any given number is a member of $A$, assuming one is allowed to use a finite number of facts regarding membership in $B$. For, if $A$ is recursive in $B$, then there are effective procedures for generating the elements of $A$ and of $\widetilde{A}$. Simply start both processes and wait until the number in question turns up in one of the lists.

As an example, let us show that for any set $B$, the set consisting of the even numbers in $B$ and the odd numbers not in $B$ is r.e. in $B$. Construct the PEFS $(E)$ as follows: Add all special axioms for $B$ (i.e., $P \bar{n}$ if $n \in B$ and $Q \bar{n}$ if 
$n \notin B$.) Let $E$ be a unary predicate different from $P$ and $Q$. Add the axioms

E0

Ex0.

Next, choose a new unary predicate $D$ and add the axioms

$D 1$

$D x 1$.

Finally, choose another new unary predicate $R$ and add the axioms

$$
\begin{aligned}
& E x \rightarrow P x \rightarrow R x \\
& D x \rightarrow Q x \rightarrow R x .
\end{aligned}
$$

It is really seen that $E$ represents (in $(E)$ ) the even natural numbers, $D$ represents (in $(E)$ ) the odd natural numbers, and $R$ represents (in $(E)$ ) the even numbers in $B$ and the odd numbers not in $B$.

For illustration, we provide a proof in $(E)$ that 4 (100 in binary) is an element of the set represented by $R$ :

$$
\begin{aligned}
& \text { P } 100 \\
& \text { E } \quad \mathrm{x} 0 \\
& \text { E } 100 \\
& E \mathrm{x} \rightarrow P x \rightarrow R x \\
& \text { E } 100 \rightarrow P 100 \rightarrow R 100 \\
& P \quad 100 \rightarrow R 100 \\
& R 100 .
\end{aligned}
$$

The following will enable us to "combine" two PEFS:

Lemma If $A$ and $B$ are sets which are representable in PEFS (E), $\left(E^{\prime}\right)$, having special axioms for $C$, then they may both be represented in a common PEFS (E") with special axioms for $C$.

Proof: Let $(E)$ and $\left(E^{\prime}\right)$ be PEFS with special axioms for $C$. Let $P, Q$ be special axiom predicates for $(E)$ and $R, S$ be those for $\left(E^{\prime}\right)$. Construct $\left(E^{\prime \prime}\right)$ as follows: Let $P, Q$ be special axiom predicates for $C$. Add the axioms $P x \rightarrow R x$ and $Q x \rightarrow S x$. $(R, S$ are assumed to be distinct from $P, Q$.) If some predicate in $(E)$ also occurs in $\left(E^{\prime}\right)$, simply replace it by a predicate new to both $(E),\left(E^{\prime}\right)$. If another predicate occurs in both systems, replace it by yet a different predicate from any used. Then collect all axioms of $(E)$ with all axioms of $\left(E^{\prime}\right)$, replacing any common predicates. This new system $\left(E^{\prime \prime}\right)$ is seen to suffice to represent both $A$ and $B$.

From now on, we will refer to this method as "rewriting predicates".

We now use common representability to exhibit a proof of the following via PEFS:

If $A$ is recursive in $B$ and $B$ is recursive in $C$, then $A$ is recursive in $C$.

Proof: Since $A$ is recursive in $B$, both $A$ and $\tilde{A}$ are r.e. in $B$; hence each of $A$, $\tilde{A}$ is representable in pseudoelementary formal systems with special axioms for $B$. By the above lemma, both $A$ and $\widetilde{A}$ are representable in a single PEFS $\left(E_{1}\right)$ 
with special axioms for $B$. Let those special axioms be $P \bar{n}$ if $n \in B, P^{\prime} \bar{n}$ if $n \notin B$. Let $Q, Q^{\prime}$ represent $A, \tilde{A}$, respectively, in $(E)$. Similarly, both $B$ and $\widetilde{B}$ are representable (by $S, S^{\prime}$ ) in a PEFS $\left(E_{2}\right)$ with special axioms $R, R^{\prime}$ for $C$.

Form the PEFS $\left(E_{3}\right)$ with special axioms for $C$ as follows: Rewriting predicates, if necessary, take all axioms of $\left(E_{2}\right)$, all regular axioms of $\left(E_{1}\right)$, along with the following axioms:

$$
\begin{aligned}
& S x \rightarrow P x \\
& S^{\prime} x \rightarrow P^{\prime} x .
\end{aligned}
$$

It is clear that $A, \tilde{A}$ are representable in $\left(E_{3}\right)$ by $Q, Q^{\prime}$, respectively.

3 Equivalence of our definition of 'r.e. in $A$ ' and 'recursive in $A$ ' with a standard formulation The formulations of 'r.e. in $A$ ' and 'recursive in $A$ ' which we show ours equivalent to are the ones in Davis [1].

A function is partial recursive in $A$ (partial $A$-recursive) if it can be obtained by a finite number of applications of composition and minimalization, beginning with the following functions:

(1) $C_{A}(x)$, the characteristic function of $A$

(2) $S(x)=x+1$

(3) $U_{i}^{n}\left(x_{1}, \ldots, x_{n}\right)=x_{i}, n \geqslant 1,1 \leqslant i \leqslant n$

(4) $x+y$

(5) $x \dot{-} y$

(6) $x \cdot y$.

A total function $f\left(x, y_{1}, \ldots, y_{n}\right)$ is called regular if $(\mu x)\left[f\left(x, y_{1}, \ldots, y_{n}\right)=0\right]$ is total. A function is recursive in $A$ ( $A$-recursive) if it can be obtained from the above list by a finite number of applications of composition and minimalization of regular functions.

A set $A$ is r.e. in $B$ if either $A=\phi$ or $A$ is the range of a function which is recursive in $B$.

A set $A$ is recursive in $B$ if $C_{A}(x)$ is a function which is recursive in $B$.

We shall need the following:

Lemma If the relation $f\left(x_{1}, \ldots, x_{n}\right)=y$ is r.e. in $B$, then the relation $f\left(x_{1}, \ldots, x_{n}\right)=y$ is recursive in $B$ (i.e., $f$ is a B-recursive function).

Proof: Let $(E)$ be a PEFS with special axioms for $B$. Let $f\left(x_{1}, \ldots, x_{n}\right)=y$ be represented by $R x_{1}, \ldots, x_{n}, y$. It is simple to show that the relation $x \neq y$ is r.e. in $B$. Let $D x, y$ represent $x \neq y$ in $(E)$. Take a new predicate $P$ and add the axiom:

$$
R x_{1}, \ldots, x_{n}, y \rightarrow D z, y \rightarrow P x_{1}, \ldots, x_{n}, z .
$$

$P$ represents $\tilde{R}$ in $(E)$. Thus, $f\left(x_{1}, \ldots, x_{n}\right)=y$ is recursive in $B$.

Theorem If $A$ is r.e. in $B$ in the standard sense, then $A$ is r.e. in $B$ in the sense of PEFS.

Proof: Case I. $A=\phi$. Construct the PEFS $(E)$ with special axioms for $B$ as follows: Take all special axioms for $B$. Let $R$ be a unary predicate not occurring in the special axioms for $B$. Then $R$ represents $\phi$ in $(E)$. Vacuously, $n \in \phi \Rightarrow$ 
$\vdash_{(E)} R \bar{n}$. Since $\bigvee_{(E)} R \bar{n}$ for all $n, \bigvee_{(E)} R \bar{n} \Rightarrow n \in \phi$. Therefore $n \in \phi \Longleftrightarrow ケ_{(E)} R \bar{n}$. Hence, $\phi$ is r.e. in $B$.

Case II: $A$ is the range of a function which is recursive in $B$. We proceed by induction, showing these functions are recursive in the sense of PEFS.

(1) $f(x)=C_{B}(x)$.

Let $P \bar{n}$ if $n \in B, Q \bar{n}$ if $n \notin B$ be the special axioms for $B$. To these, add the axioms

$$
\begin{aligned}
& P_{\bar{n}} \rightarrow C \bar{n}, 0 \\
& Q \bar{n} \rightarrow C \bar{n}, 1 .
\end{aligned}
$$

In the PEFS these axioms determine, $C x, y$ represents the relation $C_{B}(x)=y$. Therefore, the function $C_{B}(x)$ is representable in this PEFS. Hence, $C_{B}(x)$ is recursive in $B$ by our lemma.

(2) $f(x)=S(x)$.

The relation $S(x)=y$ is represented by the predicate $S$ in the EFS $(E)$ whose axioms are:

$S 0,1$

$S 1,10$

$S x 0, x 1$

$S x, y \rightarrow S x 1, y 0$.

Thus, $S(x)$ is r.e. Therefore, $S(x)$ is recursive in $B$.

(3) $f\left(x_{1}, \ldots, x_{n}\right)=U_{i}^{n}\left(x_{1}, \ldots, x_{n}\right)$ is represented by $U$ in the EFS $(E)$ with the following axiom:

$$
U x_{1}, \ldots, x_{n}, x_{i} .
$$

Thus, $U_{i}^{n}\left(x_{1}, \ldots, x_{n}\right)$ is r.e., therefore recursive in $B$.

(4) $f(x, y)=x+y$.

The relation $x+y=z$ is represented by $P$ in the EFS $(E)$ having the following axioms:

$$
P x, 0, x
$$

$S y, z \rightarrow P x, y, w \rightarrow S w, v \rightarrow P x, z, v$.

(Here, $S$ represents the successor relation.)

(5) $f(x, y)=x \dot{-} y$.

First define the function

$$
\delta(x)=\left\{\begin{array}{cl}
x-1 & \text { if } x>0 \\
0 & \text { if } x=0
\end{array}\right.
$$

The relation $\delta(x)=y$ is represented by $D$ in the EFS $(E)$ having the following axioms: 
$D 0,0$

$S x, y \rightarrow D y, x$.

The function $x \dot{-} y$ is given by

$$
x-y=\left\{\begin{array}{cl}
x-y & \text { if } x \geqslant y \\
0 & \text { if } x<y .
\end{array}\right.
$$

The relation $x-y=z$ is represented by $L$ in the $\operatorname{EFS}(E)$ having the following axioms:

$L x, 0, x$

Sy, $z \rightarrow L x, y, w \rightarrow D w, v \rightarrow L x, z, v$.

(Here, $D$ represents the function $\delta(x)$ and $S$ represents the successor relation.)

(6) $f(x, y)=x \cdot y$.

The relation $x \cdot y=z$ is represented by $M$ in the EFS $(E)$ having the following axioms:

$M x, 0,0$

$S y, z \rightarrow M x, y, w \rightarrow P w, x, v \rightarrow M x, z, v$.

$(S, P$ are as above.)

Now suppose that $f\left(x_{1}, \ldots, x_{n}\right)=g\left(h_{1}\left(x_{1}, \ldots, x_{n}\right), \ldots, h_{m}\left(x_{1}, \ldots, x_{n}\right)\right)$, where $h_{i}\left(x_{1}, \ldots, x_{n}\right)(1 \leqslant i \leqslant m)$ and $g\left(x_{1}, \ldots, x_{m}\right)$ are recursive in $B$. Then $h_{i}\left(x_{1}, \ldots, x_{n}\right)(1 \leqslant i \leqslant m)$ is representable (as a relation) by $H_{i}$ in the PEFS $\left(E_{i}\right)$, and $g\left(x_{1}, \ldots, x_{m}\right)$ is representable by $G$ in some PEFS $(E)$. Thus, $h_{i}(1 \leqslant i \leqslant m)$ and $g$ are all representable in a common PEFS $\left(E^{\prime}\right)$. Form the PEFS $\left(E^{\prime \prime}\right)$ by adding to the axioms of $\left(E^{\prime}\right)$, the following axiom:

$H_{1} x_{1}, \ldots, x_{n}, y_{1} \rightarrow \ldots \rightarrow H_{m} x_{1}, \ldots, x_{n}, y_{n} \rightarrow G y_{1}, \ldots, y_{m}, y \rightarrow F x_{1}, \ldots, x_{n}, y$.

$F$ represents the relation $f\left(x_{1}, \ldots, x_{n}\right)=y$ in $\left(E^{\prime \prime}\right)$.

Finally, assume that $f\left(x_{1}, \ldots, x_{n}\right)=(\mu y)\left[g\left(y, x_{1}, \ldots, x_{n}\right)=0\right]$, where $g\left(y, x_{1}, \ldots, x_{n}\right)$ is regular and recursive in $B$. Let $(E)$ be a PEFS in which $g\left(y, x_{1}, \ldots, x_{n}\right)$ is represented by $G$. Let $L$ represent the relation $x<y$ and $N$ represent the relation $x \neq 0$. Then construct an extension of $(E)$ by adding axioms for $L, N$, and the following:

$$
\begin{aligned}
& G 0, x_{1}, \ldots, x_{n}, 0 \rightarrow F x_{1}, \ldots, x_{n}, 0 \\
& S y, z \rightarrow G z, x_{1}, \ldots, x_{n}, 0 \rightarrow G 0, x_{1}, \ldots, x_{n}, v_{0} \rightarrow N v_{0} \rightarrow G 1, x_{1}, \ldots, x_{n}, v_{1} \rightarrow \\
& N v_{1} \rightarrow \ldots \rightarrow G y, x_{1}, \ldots, x_{n}, v_{y} \rightarrow N v_{y} \rightarrow F x_{1}, \ldots, x_{n}, z .
\end{aligned}
$$

In this PEFS, $F$ represents the relation $f\left(x_{1}, \ldots, x_{n}\right)=y$. Thus, a function recursive in $B$ in the standard sense, is recursive in $B$ via PEFS. Hence, if $A$ is r.e. in $B$ in the standard sense, then $A$ is r.e. in $B$ in the sense of PEFS.

Now suppose that $A$ is recursive in $B$ in the standard sense; i.e., $C_{A}(x)$ is recursive in $B$. This is equivalent to $A$ r.e. in $B$ and $\tilde{A}$ r.e. in $B$ (in the standard sense). But this is equivalent to $A$ r.e. in $B$ and $\tilde{A}$ r.e. in $B$ in the sense of PEFS. Hence, $A$ is recursive in $B$ in the sense of PEFS. 
We now show the converse, i.e., that $A$ is r.e. in $B$ ( $A$ is recursive in $B$ ) in the sense of PEFS implies $A$ is r.e. in $B$ ( $A$ is recursive in $B$ ) in the standard sense. To accomplish this, we arithmetize PEFS $(E)$ with special axioms for $B$. Our method of arithmetization is similar to that of Mendelson [3].

Let $(E)$ be a PEFS over $\{0,1\}$ with special axioms for $B$. Let $P_{n}^{m}$ be the $n^{\text {th }} m$-place predicate letter. We assign Gödel numbers to the symbols as follows:

$$
\begin{aligned}
& g n(0)=3, g n(1)=5, g n(\rightarrow)=7, g n(,)=9 . \\
& g n\left(x_{i}\right)=7+4 i, i \geqslant 1 . \\
& g n\left(P_{n}^{m}\right)=9+4\left(2^{m} \cdot 3^{n}\right), m, n \geqslant 1 .
\end{aligned}
$$

Now let $M$ be the expression $\alpha_{1} \alpha_{2} \ldots \alpha_{n}$, where each $\alpha_{i}$ is a symbol. Let $a_{1}, \ldots, a_{n}$ be the corresponding Gödel numbers of these symbols. Then we define the Gödel number of $M$ as follows:

$$
g n(M)=\prod_{k=1}^{n} \operatorname{Pr}_{k}^{a_{k}}
$$

where $P r_{k}$ is the $k^{\text {th }}$ prime number, with $P r_{1}=2$.

Let $M_{1}, \ldots, M_{n}$ be a finite sequence of expressions. Then we define the Gödel number of this sequence as follows:

$$
g n\left(M_{1}, \ldots, M_{n}\right)=\prod_{k=1}^{n} \operatorname{Pr}_{k}^{g n\left(M_{k}\right)}
$$

For the remainder of this section, we assume familiarity with basic relations and functions which are recursive. The reader is referred to Mendelson [3] or Davis [1] for detailed information.

We will need the following functions and relations. These are all recursive.

(i) $x \mid y$ holds if and only if $x$ divides $y$.

(ii) $(x)_{n}=(\mu y)_{\leqslant x}\left[\operatorname{Pr}_{n}^{y} \mid x \wedge \sim\left(\operatorname{Pr}_{n}^{y+1} \mid x\right)\right]$.

(iii) $q t(x, y)$ is the quotient upon division of $y$ by $x$.

(iv) $L(y)$ is the number of nonzero exponents in the prime factorization of $x$.

(v) $G N(x)$ holds if and only if there exist positive integers $a_{k}, 1 \leqslant k \leqslant n$, such that $x=\prod_{k=1}^{n} \operatorname{Pr}_{k}^{a_{k}}$.

(vi) Term $(x, z)$ holds if and only if $z=\prod_{k=1}^{n} \operatorname{Pr}_{k}^{a_{k}}$ for suitable $a_{k}>0$ and $x=a_{k}$ for some $k, 1 \leqslant k \leqslant n$.

(vii) $x * y=x \prod_{i=0}^{L(y) \dot{-1}} \operatorname{Pr}_{L(x)+i+1}^{(y)_{i+1}}$.

If $M, N$ are expressions, then $g n(M N)=g n(M) * g n(N)$.

We now show the following functions and relations to be recursive (recursive in $B$ if followed by a subscript $B$ ). 
(1) $V(x)$ holds iff $x$ is the Gödel number of an expression consisting of a variable.

$$
V(x) \Longleftrightarrow(\exists y \leqslant x)\left(1 \leqslant y \wedge x=2^{7+4 y}\right) .
$$

(2) Pred(x) holds iff $x$ is the Gödel number of an expression consisting of a predicate letter.

$$
\operatorname{Pred}(x) \Longleftrightarrow(\exists y \leqslant x)(\exists z \leqslant x)\left[1 \leqslant y \wedge 1 \leqslant z \wedge x=2^{9+4\left(2^{y} \cdot 3^{z}\right)}\right] .
$$

(3) $\operatorname{Trm}(x)$ holds iff $x$ is the Gödel number of a term.

$$
\begin{array}{r}
\operatorname{Trm}(x) \Longleftrightarrow G N(x) \wedge(\forall y \leqslant x)[\operatorname{Term}(y, x) \Rightarrow(y=3 \vee y=5 \vee \\
(\exists z \leqslant y)(y=7+4 z \wedge 1 \leqslant z))] .
\end{array}
$$

(4) $\operatorname{Arg}(x)$ : If $x$ is the Gödel number of a predicate letter $P_{n}^{m}$, then $\operatorname{Arg}(x)=m$.

$$
\operatorname{Arg}(x)=(q t(4, x-9))_{1} .
$$

(5) At Form ( $x$ ) holds iff $x$ is the Gödel number of an atomic formula. This holds if and only if there are terms $t_{1}, \ldots, t_{n}$ and a predicate letter $P_{n}^{m}$ such that $x$ is the Gödel number of $P_{n}^{m} t_{1}, \ldots, t_{n}$. This holds if and only if there is a sequence of expressions

$$
P_{n}^{m} ; P_{n}^{m} t_{1}, ; P_{n}^{m} t_{1}, t_{2}, ; \ldots ; P_{n}^{m} t_{1}, \ldots, t_{n-1}, ; P_{n}^{m} t_{1}, \ldots, t_{n-1}, t_{n} .
$$

This sequence of $n+1$ expressions can be represented by its Gödel number $y$. We have $y<\operatorname{Pr}_{n+1}^{x}<\operatorname{Pr}_{x}^{x}$. Also note that $n=\operatorname{Arg}\left((x)_{1}\right)$.

$$
\begin{gathered}
\text { At Form }(x) \Longleftrightarrow\left(\exists y<\operatorname{Pr}_{x}^{x}\right)\left[x=(y)_{L(y)} \wedge \operatorname{Pred}\left((y)_{1}\right) \wedge L(y)=\operatorname{Arg}\left((x)_{1}\right)+1 \wedge\right. \\
(\forall u<L(y))\left(\left(u>1 \wedge u \leqslant \operatorname{Arg}\left((x)_{1}\right)\right) \Rightarrow\right. \\
\left.\left.(\exists v<y)\left((y)_{u}=(y)_{u-1} * v * 2^{9} \wedge \operatorname{Trm}(v)\right)\right)\right] .
\end{gathered}
$$

(6) Form (x) holds iff $x$ is the Gödel number of a formula.

$$
\begin{gathered}
\text { Form }(x) \Longleftrightarrow \operatorname{At~Form~}(x) \vee(\exists y<x)(\exists z<x)[\text { Form }(y) \wedge \text { Form }(z) \wedge \\
\left.x=y * 2^{7} * z\right] .
\end{gathered}
$$

(7) $M P(x, y, z)$ holds iff the formula with Gödel number $z$ is a direct consequence of the formulas with Gödel number $x$ and Gödel number $y$ by modus ponens.

$$
M P(x, y, z) \Longleftrightarrow \text { At Form }(x) \wedge \text { Form }(y) \wedge \text { Form }(z) \wedge y=x * 2^{7} * z
$$

(8) Word $(x)$ holds iff $x$ is the Gödel number of a word in $K=\{0,1\}$.

$$
\text { Word }(x) \Longleftrightarrow x=2^{3} \vee x=2^{5} \vee(\exists y<x)\left[\left(\text { Word }(y) \wedge\left(x=y * 2^{3} \vee x=y * 2^{5}\right)\right]\right.
$$

(9) $\operatorname{Sub}(x, y, z, w)$ holds iff $w$ is the Gödel number of the expression resulting from substituting the word in $\{0,1\}$ with Gödel number $z$ for all occurrences of the variable with Gödel number $y$ in the formula with Gödel number $x$. 


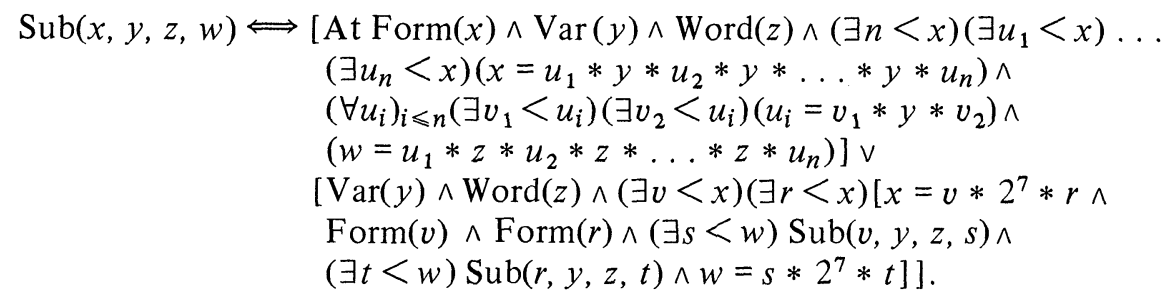

(10) $\operatorname{Reg} A x(x)$ holds iff $x$ is the Gödel number of a regular axiom. We assume this relation to be recursive, i.e., we can effectively recognize whether or not a given number is the Gödel number of an axiom.

(11) $\operatorname{Spec} A x_{\mathrm{B}}(x)$ holds iff $x$ is the Gödel number of a special axiom for the set $B$. We want to say that there are predicates $P, Q$ such that $X \in B$ implies $P X$ is an axiom and $X \notin B$ implies $Q X$ is an axiom. Let us suppose, for definiteness, that $P$ is $P_{1}^{1}$ and $Q$ is $P_{2}^{1}$.

$$
\begin{gathered}
\operatorname{Spec} A x_{B}(x) \Longleftrightarrow(\forall y<x)\left[\operatorname { W o r d } ( y ) \Rightarrow \left[\left(C_{B}(y)=0 \wedge x=2^{7+4\left(2^{1} \cdot 3^{1}\right)} \cdot 3^{g n(y)}\right) \vee\right.\right. \\
\left.\left.\left(C_{B}(y)=1 \wedge x=2^{7+4\left(2^{1} \cdot 3^{2}\right)} \cdot 3^{g n(y)}\right)\right]\right] .
\end{gathered}
$$

Note that there are an infinite number of special axioms for $B$; one for each $n \in \mathbf{N}$.

$(12)_{\mathrm{B}} A x(x)$ holds iff $x$ is the Gödel number of an axiom of $(E)$.

$$
A x(x) \Longleftrightarrow \operatorname{Reg} A x(x) \vee \operatorname{Spec} A x(x) .
$$

$(13)_{B}$ PEFS $(x)$ holds iff $x$ is the Gödel number of a pseudoelementary formal system.

$$
\operatorname{PEFS}(x) \Longleftrightarrow(\forall y \leqslant L(x))\left(A x\left((x)_{y}\right)\right) .
$$

(14) $\mathrm{B} P f_{B}(x)$ holds iff $x$ is the Gödel number of a proof in a PEFS $(E)$ with special axioms for $B$.

$$
\begin{aligned}
P f(x) \Longleftrightarrow & \operatorname{PEFS}(x) \wedge(\exists y<x)\left(x=2^{y} \wedge A x(y)\right) \vee \\
& (\exists y<x)(\exists z<y)(\exists w<x)\left[\operatorname{Sub}(y, z, w, x) \wedge P f_{B}(y)\right] \vee \\
& (\exists y<x)(\exists z<x)\left[M P(y, z, x) \wedge P f_{B}(y) \wedge P f_{B}(z)\right] .
\end{aligned}
$$

(15) $P f_{B}(y, x)$ holds iff $y$ is the Gödel number of a proof of the expression with Gödel number $x$, in a PEFS $(E)$ with special axioms for $B$.

$$
P f_{B}(y, x) \Longleftrightarrow P f_{B}(y) \wedge x=(y)_{L(y)} \text {. }
$$

Suppose now that $A$ is representable in the PEFS $(E)$ with special axioms for $B$. Then for some predicate letter, say $P_{i}^{1}, \vdash_{(E)} P_{i}^{1} \bar{n} \Longleftrightarrow n \epsilon A$, where $\bar{n}$ is $n$ in binary notation ( $\bar{x}$ is recursive). Thus

$$
x \in A \Longleftrightarrow \vdash_{(E)} P_{i}^{1} \bar{x} \Longleftrightarrow(\exists y) P f_{B}\left(y, 2^{9+4\left(2^{1} \cdot 3^{i}\right)} * g n(\bar{x})\right) .
$$

Therefore, $A$ is r.e. in $B$ in the standard sense. We have now shown the following:

Theorem $A$ is r.e. in $B$ in the sense of PEFS implies $A$ is r.e. in $B$ in the standard sense. 
Corollary $\quad A$ is recursive in $B$ in the sense of PEFS implies $A$ is recursive in $B$ in the standard sense.

Thus the notions of 'r.e. in' and 'recursive in' as defined via PEFS are equivalent to the standard notions. Similar results follow for relations and functions.

4 Basic theorems of relative recursion theory In this section, we show how to prove some basic recursion theorems in the setting of PEFS.

We first note that in referring to the Gödel number of a PEFS $(E)$ with special axioms for $B$, we don't have to include the special axioms in the $g n$. $e$. Since all such systems have the same special axioms, it is superfluous. Besides, they form an infinite set.

Let the PEFS $(E)$ consist of the sequence of axioms $A_{1}, \ldots, A_{n}$ such that $A_{n}$ is of the form $P_{n_{1}} \rightarrow \ldots \rightarrow P_{n_{k}}$. The predicate letter $P_{n_{k}}$ is called the principal predicate of $(E)$. The reason we use principal predicates is that in a given PEFS, more than one relation may be representable. Given the Godel number of a PEFS, we want to be able to determine which relation is signified. This may be done by looking at the principal predicate.

$(16)_{\mathrm{B}} \operatorname{Ded}_{B}(z, y)$ iff $z$ is the Gödel number of a PEFS $(E)$ with special axioms for $B$, and $y$ is the Gödel number of a proof from $(E)$.

$$
\begin{aligned}
\operatorname{Ded}_{B}(z, y) \Longleftrightarrow & \operatorname{PEFS}(z) \wedge(\forall x \leqslant L(y))\left[( \exists w \leqslant L ( z ) ) \left((z)_{w}=\right.\right. \\
& \left.(z)_{x}\right) \vee(\exists u<x)(\exists v<x) M P\left((z)_{u},(z)_{v},(z)_{x}\right) \vee \\
& \left.(\exists u<x)(\exists v<x)(\exists w<x) \operatorname{Sub}\left((z)_{u},(z)_{v},(z)_{w},(z)_{x}\right)\right] .
\end{aligned}
$$

$(17)_{\mathrm{B}} S_{n}^{B}\left(z, x_{1}, \ldots, x_{n}, y\right)$ iff $z$ is the Gödel number of a binary PEFS $(E)$ with special axioms for $B$, having $P_{i}^{n}$ as its principal predicate and $y$ is the Gödel number of a proof in $(E)$ of $P_{i}^{n} \bar{x}_{1}, \ldots, \bar{x}_{n}$, where $\bar{x}$ is $x$ in binary notation.

$$
\begin{aligned}
S_{n}^{B}\left(z, x_{1}, \ldots, x_{n}, y\right) \Longleftrightarrow & \operatorname{Ded}_{B}(z, y) \wedge \operatorname{Arg}\left(\left((y)_{L(y)}\right)_{1}\right)=n \wedge\left((y)_{L(y)}\right)_{1}= \\
& \left.\left(\left((z)_{L(z)}\right)_{L((z)} L(z)\right)\right)_{1} \wedge(\exists w<y)\left[(y)_{L(y)}=\right. \\
& \left.2^{9+4\left(2^{n} \cdot 3^{w}\right)} * g n\left(\bar{x}_{1}\right) * 2^{9} * g n\left(\bar{x}_{2}\right) * 2^{9} * \ldots * 2^{9} * g n\left(\bar{x}_{n}\right)\right] .
\end{aligned}
$$

(18) $U(x)=(\mu y<x)\left[\bar{y}=\left((x)_{L(x)}\right)_{L\left((x)_{L(x)}\right)}\right]$.

If $x$ is the Gödel number of a proof of $P_{i}^{n} \bar{x}_{1}, \ldots, \bar{x}_{n}$, then $U(x)=x_{n}$.

Now suppose that $f^{B}\left(x_{1}, \ldots, x_{n}\right)$ is an $n$-ary $B$-partial recursive function.

Then $f^{B}\left(x_{1}, \ldots, x_{n}\right)=x$ is an $(n+1)$-ary $B$-partial recursive relation. For this relation to be representable in a PEFS $(E)$ with special axioms for $B$, there must be a predicate letter $P_{i}^{n+1}$ of $(E)$ such that $f^{B}\left(k_{1}, \ldots, k_{n}\right)=k \Longleftrightarrow \vdash_{(E)} P_{i}^{n+1} \bar{k}_{1}, \ldots$, $\bar{k}_{n}, \bar{k}$ for all natural numbers $k_{1}, \ldots, k_{n}, k$.

Normal Form Theorem There exists a recursive function $\bar{U}$ and a $B$-recursive function $\bar{S}_{n}^{B}$ such that if $f^{B}\left(x_{1}, \ldots, x_{n}\right)$ is a partial $B$-recursive function, which, as a relation, is representable in the PEFS $(E)$ having special axioms for $B$ and Gödel number e, then for all $x_{1}, \ldots, x_{n}$ in $\mathbf{N}$,

$$
f^{B}\left(x_{1}, \ldots, x_{n}\right) \simeq \bar{U}\left((\mu w) \bar{S}_{n}^{B}\left(e, x_{1}, \ldots, x_{n}, w\right)\right) .
$$


Proof: $f^{B}\left(k_{1}, \ldots, k_{n}\right)=k \Longleftrightarrow \vdash_{(E)} P_{i}^{n+1} \bar{k}_{1}, \ldots, \bar{k}_{n}, \bar{k}$, where $P_{i}^{n+1}$ is the principal predicate letter of $(E)$. Now $f^{B}\left(k_{1}, \ldots, k_{n}\right)$ is defined $\Longleftrightarrow(\exists x)(\exists y) S_{n+1}^{B}(e$, $\left.k_{1}, \ldots, k_{n}, x, y\right) \Longleftrightarrow(\exists w) S_{n+1}^{B}\left(e, k_{1}, \ldots, k_{n},(w)_{0},(w)_{1}\right)$. Define $\bar{S}_{n}^{B}\left(e, k_{1}, \ldots\right.$, $\left.k_{n}, w\right)=S_{n+1}^{B}\left(e, k_{1}, \ldots, k_{n},(w)_{0},(w)_{1}\right)$. Therefore, $f^{B}\left(k_{1}, \ldots, k_{n}\right)$ is defined $\Longleftrightarrow(\exists w) \bar{S}_{n}^{B}\left(e, k_{1}, \ldots, k_{n}, w\right)$. If $f\left(k_{1}, \ldots, k_{n}\right)$ is defined, then $\left((\mu w) \bar{S}_{n}^{B}(e\right.$, $\left.\left.k_{1}, \ldots, k_{n}, w\right)\right)_{1}$ is the Gödel number of a proof in $(E)((E)$ has special axioms for $B)$ of $P_{i}^{n+1} \bar{k}_{1}, \ldots, \bar{k}_{n}, \bar{k}$. Therefore, $U\left(\left((\mu w) \bar{S}_{n}^{B}\left(e, k_{1}, \ldots, k_{n}, w\right)\right)_{1}\right)=$ $f^{B}\left(k_{1}, \ldots, k_{n}\right)$. Let $\bar{U}(x)=U\left((x)_{1}\right)$. Thus $f^{B}\left(x_{1}, \ldots, x_{n}\right) \simeq \bar{U}\left((\mu w) \vec{S}_{n}^{B}\left(e, x_{1}, \ldots\right.\right.$, $\left.x_{n}, w\right)$ ). It is obvious that $\bar{S}_{n}^{B}$ is recursive in $B$ and $\bar{U}$ is recursive.

Lemma If $R\left(x_{1}, \ldots, x_{n}, y\right)$ is a relation which is recursive in $B$, then for some $e,(\exists y) R\left(x_{1}, \ldots, x_{n}, y\right) \Longleftrightarrow(\exists y) \bar{S}_{n}^{B}\left(e, x_{1}, \ldots, x_{n}, y\right)$.

Proof: Let $C_{R}\left(x_{1}, \ldots, x_{n}, y\right)$ be the characteristic function of the $B$-recursive relation $R\left(x_{1}, \ldots, x_{n}, y\right)$. Then $C_{R}\left(x_{1}, \ldots, x_{n}, y\right)$ is recursive in $B$. Thus the function $(\mu y)\left(C_{R}\left(x_{1}, \ldots, x_{n}, y\right)=0\right)$ is partial recursive in $B$. Let $e$ be the Gödel number of a PEFS $(E)$ in which $(\mu y)\left(C_{R}\left(x_{1}, \ldots, x_{n}, y\right)=0\right)=z$ is representable. Now, $(\exists y) R\left(x_{1}, \ldots, x_{n}, y\right) \Longleftrightarrow(\mu y)\left(C_{R}\left(x_{1}, \ldots, x_{n}, y\right)=0\right)$ is defined. Therefore, $(\exists y) R\left(x_{1}, \ldots ., x_{n}, y\right) \Longleftrightarrow(\exists z)(\exists w) S_{n+1}^{B}\left(e, x_{1}, \ldots, x_{n}, z, w\right) \Longleftrightarrow$ $(\exists v) S_{n+1}^{B}\left(e, x_{1}, \ldots, x_{n},(v)_{0},(v)_{1}\right) \Longleftrightarrow(\exists v) \bar{S}_{n}^{B}\left(e, x_{1}, \ldots, x_{n}, v\right)$.

Lemma $A$ relation $R\left(x_{1}, \ldots, x_{n}\right)$ is r.e. in $B \Longleftrightarrow\left(x_{1}, \ldots, x_{n}\right) \in R$ is expressible in the form ( $\exists y) P\left(x_{1}, \ldots, x_{n}, y\right)$, where $P$ is recursive in $B$.

Proof: Suppose $R\left(x_{1}, \ldots, x_{n}\right)$ is r.e. in $B$. Then for some PEFS $(E)$ with Gödel number $e$, and some predicate $P_{i}^{n}, R\left(m_{1}, \ldots, m_{n}\right) \Longleftrightarrow \vdash_{(E)} P_{i}^{n} \bar{m}_{1}, \ldots, \bar{m}_{n} \Longleftrightarrow$ ( $\exists y) S_{n}^{B}\left(e, m_{1}, \ldots, m_{n}, y\right)$.

Conversely, suppose that $\left(x_{1}, \ldots, x_{n}\right) \in R$ is expressible in the form ( $\exists y) P\left(x_{1}, \ldots, x_{n}, y\right)$, where $P$ is recursive in $B$. Let $P$ represent $P$ in some PEFS $(E)$ with special axioms for $B$. Let $T$ be a predicate letter not occurring in $(E)$. Add the following axiom to $(E)$, obtaining $\left(E^{\prime}\right)$ :

$$
\bar{P} x_{1}, \ldots, x_{n}, y \rightarrow T x_{1}, \ldots, x_{n} .
$$

Then $T$ represents $R\left(x_{1}, \ldots, x_{n}\right)$ in $\left(E^{\prime}\right)$. Hence, $R$ is r.e. in $B$.

Corollary $\quad A$ set $A$ is r.e. in $B \Longleftrightarrow x \in A$ is expressible in the form $(\exists y) P(x, y)$, where $P$ is recursive in $B$.

By the second lemma, if $R\left(x_{1}, \ldots, x_{n}\right)$ is r.e. in $B$, then $R\left(x_{1}, \ldots, x_{n}\right) \Longleftrightarrow$ ( $\exists y) P\left(x_{1}, \ldots, x_{n}, y\right)$, where $P$ is recursive in $B$. By the first lemma $(\exists y)$ $P\left(x_{1}, \ldots, x_{n}, y\right) \Longleftrightarrow(\exists y) \bar{S}_{n}^{B}\left(e, x_{1}, \ldots, x_{n}, y\right)$. Thus $R\left(x_{1}, \ldots, x_{n}\right) \Longleftrightarrow$ ( $\exists y) \bar{S}_{n}^{B}\left(e, x_{1}, \ldots, x_{n}, y\right)$ for some $e$. On the other hand, for each $e,(\exists y) S_{n}^{B}(e$, $\left.x_{1}, \ldots, x_{n}, y\right)$ is an $n$-place relation which is r.e. in $B$. This implies the following:

Theorem The n-ary relations which are r.e. in $B$ are precisely those which are of the form $(\exists y) \bar{S}_{n}^{B}\left(e, x_{1}, \ldots, x_{n}, y\right)$ for $e \in \mathbf{N}$.

Corollary The sets which are r.e. in $B$ are precisely those which are of the form $(\exists y) \bar{S}_{1}^{B}(e, x, y)$ for $e \in \mathbf{N}$. 
Enumeration Theorem For each $n \geqslant 1$ we can enumerate the $n$-place relations which are r.e. in $B$ in such a manner so that the relation ' $\left(m_{1}, \ldots, m_{n}\right)$ satisfies the $j^{\text {th }}$ relation which is r.e. in $B$ ' is itself r.e. in $B$.

Proof: The $n$-ary relations which are recursive in $B$ are of the form $(\exists y)$ $\bar{S}_{n}^{B}\left(e, x_{1}, \ldots, x_{n}, y\right)$. As $e$ runs through the natural numbers, we get all such relations. This relation itself is r.e. in $B$.

Corollary We can enumerate the sets which are r.e. in $B$ in such a manner so that the relation ' $i$ is an element of the $j^{\text {th }}$ set which is r.e. in $B$ ' is itself r.e. in $B$.

From now on, we will refer to the $i^{\text {th }} n$-place relation which is r.e. in $B$ by $R_{i}^{B, n}\left(x_{1}, \ldots, x_{n}\right)$. Note that $R_{i}^{B, n}\left(x_{1}, \ldots, x_{n}\right)=\left\{\left(x_{1}, \ldots, x_{n}\right) \mid(\exists y) \bar{S}_{n}^{B}\left(x_{1}, \ldots\right.\right.$, $\left.\left.x_{n}, y\right)\right\}$.

Iteration Theorem There is a recursive function $f\left(z_{1}, \ldots, z_{n}\right)$ such that for any set $B$, and any $B$-r.e. relation $R_{e}^{B, n+m}\left(z_{1}, \ldots, z_{n}, x_{1}, \ldots, x_{m}\right)$, and any $i_{1}, \ldots, i_{n}, f\left(i_{1}, \ldots, i_{n}\right)$ is an index of $R_{e}^{B, n+m}\left(i_{1}, \ldots, i_{n}, x_{1}, \ldots, x_{m}\right)$ as a relation which is r.e. in $B .\left[R_{e}^{B, n+m}\left(i_{1}, \ldots, i_{n}, x_{1}, \ldots, x_{m}\right)=R_{f\left(e, i_{1}, \ldots, i_{n}\right)}^{B, m}\left(x_{1}, \ldots, x_{n}\right)\right.$.]

Proof: That $R_{e}^{B, n+m}\left(i_{1}, \ldots, i_{n}, x_{1}, \ldots, x_{m}\right)$ is r.e. in $B$ is seen as follows: Let $(E)$ be the PEFS with special axioms for $B$ and Gödel number $e$, in which $R_{e}^{B, n+m}\left(z_{1}, \ldots, z_{n}, x_{1}, \ldots, x_{m}\right)$ is represented by $P z_{1}, \ldots, z_{n}, x_{1}, \ldots, x_{m}$. Take a new predicate $Q$ and add the following axiom to $(E)$ to get $\left(E^{\prime}\right)$ :

$$
P \bar{i}_{1}, \ldots, \bar{i}_{n}, x_{1}, \ldots, x_{m} \rightarrow Q x_{1}, \ldots, x_{m} .
$$

Then $Q$ represents $R_{e}^{B, n+m}\left(i_{1}, \ldots, i_{n}, x_{1}, \ldots, x_{m}\right)$ in this new system. Thus, $R_{e}^{B, n+m}\left(i_{1}, \ldots, i_{n}, x_{1}, \ldots, x_{m}\right)$ is r.e. in $B$.

Let $p, q$ be the Gödel numbers of $P, Q$ and let $v_{1}, \ldots, v_{m}$ be the Gödel numbers of $x_{1}, \ldots, x_{m}$. Let $\bar{n}=g n(n), c, a$ be the Gödel numbers of , and $\rightarrow$. Then $f\left(i_{1}, \ldots, i_{n}\right)=e * p * \bar{i}_{1} * c * \ldots * c * \bar{i}_{n} * c * v_{1} * c * \ldots * v_{n} * a * q *$ $v_{1} * c * \ldots * v_{m} . f\left(i_{1}, \ldots, i_{n}\right)$ is recursive and is the Gödel number of $\left(E^{\prime}\right)$, which serves to represent $R_{e}^{B, n+m}\left(i_{1}, \ldots, i_{n}, x_{1}, \ldots, x_{m}\right)$.

The reader has probably noticed that elementary formal systems provide a particularly elegant framework for recursion theoretic arguments. One reason they work more smoothly than, say, Turing machines in certain contexts is that elementary formal systems are nondeterministic. A systematic proof procedure can be specified, once and for all, independent of any particular choice of elementary formal system axiom set, which, if followed, will cause anything derivable eventually to be derived. But this is not part of the elementary formal system machinery. The elementary formal system rules are permissible, not mandatory. Thus much of the step-by-step detail work inherent in a deterministic approach is bypassed.

Another reason is that an elementary formal system is more in the spirit of a high-level programming language, as opposed to, say, Turing machines, which act more on the level of an assembly language. A few axioms of a simple elementary formal system would correspond to a large amount of code required of a Turing machine. In a high-level language, an instruction may be seen as an 
algebraic operation or as a powerful string-manipulation procedure. However, in an assembly language it is necessary painstakingly to specify on a very primitive level, working with small pieces of data at any time, just how that operation or procedure is to be carried out. This is, of course, subject to certain limitations, as certain fundamental operations and procedures are part of a real computer's hardware, and are implicit in the assembly language (and also in a high-level language). ${ }^{1}$

\section{NOTE}

1. For the interested reader, it is worth pointing out that Melvin Fitting, in his forthcoming book Fundamentals of Generalized Recursion Theory, uses elementary formal systems to provide an abstract framework for studying computational structures.

\section{REFERENCES}

[1] Davis, M., Computability and Undecidability, McGraw-Hill, New York, 1958.

[2] Horowitz, B. M., Theory of Effectively Non-Arithmetical Sets, (Ph.D. Thesis), City University of New York, 1975.

[3] Mendelson, E., Introduction to Mathematical Logic, D. Van Nostrand, Princeton, New Jersey, 1964.

[4] Smullyan, R. M., Theory of Formal Systems, Princeton University Press, Princeton, New Jersey, 1961.

Department of Mathematics

Rutgers University

Newark, New Jersey 07102 